\title{
ARTICLE
}

Received 5 Mar 2015 | Accepted 19 May 2015 | Published 30 Jun $2015 \quad$ DOl: 10.1038/ncomms8574

\section{Supramolecular amplification of amyloid self-assembly by iodination}

\author{
Arianna Bertolani ${ }^{1}$, Lisa Pirrie ${ }^{1,2}$, Loic Stefan ${ }^{1}$, Nikolay Houbenov$^{3}$, Johannes S. Haataja ${ }^{3}$, Luca Catalano ${ }^{1}$, \\ Giancarlo Terraneo ${ }^{1}$, Gabriele Giancane ${ }^{4}$, Ludovico Valli ${ }^{4}$, Roberto Milani ${ }^{2}$, Olli Ikkala ${ }^{3}$, Giuseppe Resnati ${ }^{1}$ \& \\ Pierangelo Metrangolo 1,2
}

Amyloid supramolecular assemblies have found widespread exploitation as ordered nanomaterials in a range of applications from materials science to biotechnology. New strategies are, however, required for understanding and promoting mature fibril formation from simple monomer motifs through easy and scalable processes. Noncovalent interactions are key to forming and holding the amyloid structure together. On the other hand, the halogen bond has never been used purposefully to achieve control over amyloid self-assembly. Here we show that single atom replacement of hydrogen with iodine, a halogen-bond donor, in the human calcitonin-derived amyloidogenic fragment DFNKF results in a super-gelator peptide, which forms a strong and shape-persistent hydrogel at 30-fold lower concentration than the wild-type pentapeptide. This is remarkable for such a modest perturbation in structure. Iodination of aromatic amino acids may thus develop as a general strategy for the design of new hydrogels from unprotected peptides and without using organic solvents.

\footnotetext{
${ }^{1}$ Laboratory of Nanostructured Fluorinated Materials (NFMLab), Department of Chemistry, Materials, and Chemical Engineering 'Giulio Natta', Politecnico di Milano, Via Mancinelli 7, Milano I-20131, Italy. ${ }^{2}$ VTT-Technical Research Centre of Finland, PO Box 1000, Espoo FI-02044, Finland. ${ }^{3}$ Department of Applied Physics, Aalto University, PO Box 15100, Espoo FI-02150, Finland. ${ }^{4}$ Dipartimento Beni Culturali, Università del Salento, Lecce I-73100, Italy. Correspondence and requests for materials should be addressed to P.M. (email: pierangelo.metrangolo@polimi.it).
} 
A myloid fibrils are a class of nanomaterials that can be assembled from a wide variety of peptides and proteins and have an array of functional and pathological roles in nature $^{1}$. In the latter, the self-assembly process can be triggered by peptide mutations and leads to the formation of insoluble fibrillar aggregates that are associated with Alzheimer's, Parkinson's, Creutzfeldt-Jakob and Huntington's diseases ${ }^{2-6}$. Amyloid fibrils also perform a number of physiological roles including being constituents of protective envelopes of fish and insect eggs, essential amphipathic materials of fungi and bacteria and a vital constituent of spider silk ${ }^{7}$. This wide variety of physiological functions of amyloids has inspired many nanocomposites thanks to the robustness of these fibrillar materials, which are comparable to dragline silk and collagen, as well as biomaterial applications like tissue engineering ${ }^{8-11}$. In this context, it is important that the amyloidogenic peptide/protein self-assembles in a tunable and controlled manner to provide fibrils of well-defined architecture ${ }^{12}$.

Amyloid fibrils are characterized by their cross- $\beta$-sheet structures whereby bundles of $\beta$-strands form highly ordered filaments that run perpendicular to the fibre axis ${ }^{11,13}$. The stability of amyloid structures can be attributed to noncovalent interactions, namely hydrogen bonds, $\pi-\pi$ stacking and hydrophobic interactions occurring between both side chain and backbone atoms, which hold the $\beta$-sheets and strands together ${ }^{11}$. On the basis of the assumption that noncovalent interactions are key to the formation of the amyloid structure, and since halogen bonds have been recently appreciated as new tools for supramolecular engineering ${ }^{14}$, we decided to study the impact of introducing halogen-bond donor groups, for example, iodine atoms, into an already known amyloidogenic motif to study the potential of halogen bonding in controlling and promoting amyloid self-assembly.

Although many modifications of amyloidogenic sequences have been utilized to tune their self-assembly behaviour ${ }^{12}$, halogenation has rarely been pursued and only on single amino acids or on a dipeptide ${ }^{15-17}$. The advantage of a strategy based on the introduction of halogen atoms on amyloidogenic motifs lies in the fact that halogenation is a minimal structural modification, which, on the other hand, may induce a large difference in the peptide supramolecular behaviour as a consequence of the rich variety of noncovalent interactions given by halogen atoms ${ }^{18}$. It has been shown previously that incorporation of single halogen substituents on the aromatic side chain of amino acids enhances their self-assembly into amyloid-like fibrils that promote hydrogelation in aqueous solvents ${ }^{19}$. In particular, fluorinated amino acids were found to function better than chlorinated and brominated analogues in terms of assembly kinetics and rigidity of the obtained hydrogels. This has been explained by the significant electronic effects of fluorine and the subsequent perturbation of the energetics of the $\pi-\pi$ stacking interactions, although hydrophobic and steric effects could not be discounted $^{20}$. Conversely, there is only one report on the fibrillar nanostructures given by a diiodinated dipeptide ${ }^{17}$ but, to the best of our knowledge, no detailed study on the effect of iodination on promoting/inhibiting the self-assembly of amyloidogenic peptides. Under the hypothesis that introducing an iodine atom on the $p$-position of the benzyl side chain of an aromatic amino acid may result in the formation of halogen bonding thus promoting fibrils' formation, we studied the structure of $p$-iodo-phenylalanine ( $p$-I-Phe) and the selfassembly behaviour of a series of iodinated derivatives of a known amyloidogenic core sequence.

\section{Results}

Single crystal $\mathrm{X}$-ray structure of $\boldsymbol{p}$-iodo-phenylalanine. The halogen bond ${ }^{21}$ is the strong, specific and directional interaction involving halogen atoms as electrophilic species ${ }^{22}$. Increasing in strength with halogen atom polarizability, halogen bonds are most efficient with iodine substituents. This rather new interaction is virtually unexplored in the field of amino acid and peptide self-assembly. For this reason, to evaluate the possibility of exploiting the halogen bond in the self-assembly of amyloid peptides/proteins, we have first studied the single crystal X-ray structure of the amino acid $p$-I-Phe.

The crystal structure of $p$-I-Phe (Supplementary Fig. 1 and Supplementary Table 1) highlights the amphoteric behaviour of the iodine atom, which functions as Lewis acid along the extension of the C-I bond and as Lewis base at the belt perpendicular to it (Supplementary Fig. 2a). In fact, the crystal packing of this iodinated amino acid is characterized by two strands of amino acids running along the $b$ crystallographic axis and connected through hydrogen bonds (Supplementary Fig. 2b). These strands are then laterally assembled into two-dimensional supramolecular sheets thanks to type II iodine... iodine contacts $^{23}$, that is, halogen bonds (I . . I I distance 3.7517(4) $\AA$, I ... I. I angle $89.66^{\circ}$ ), occurring at the strand surface (Supplementary Fig. 2c). Importantly, no sign of $\pi-\pi$ stacking is observed, while $\mathrm{C}-\mathrm{H} \cdots \pi$ interactions involving the benzyl hydrogens contribute to aromatic side-chain ordering. Despite the fact that inferring structural information on the amyloid state from high-resolution X-ray diffraction analysis of small-molecule models is difficult, we introduced the $p$-I-Phe residue into the amyloidogenic core sequence DFNKF ${ }^{24}\left(\mathrm{NH}_{2}\right.$-Asp-Phe-Asn-LysPhe-COOH, Fig. 1), with the purpose of exploiting similar

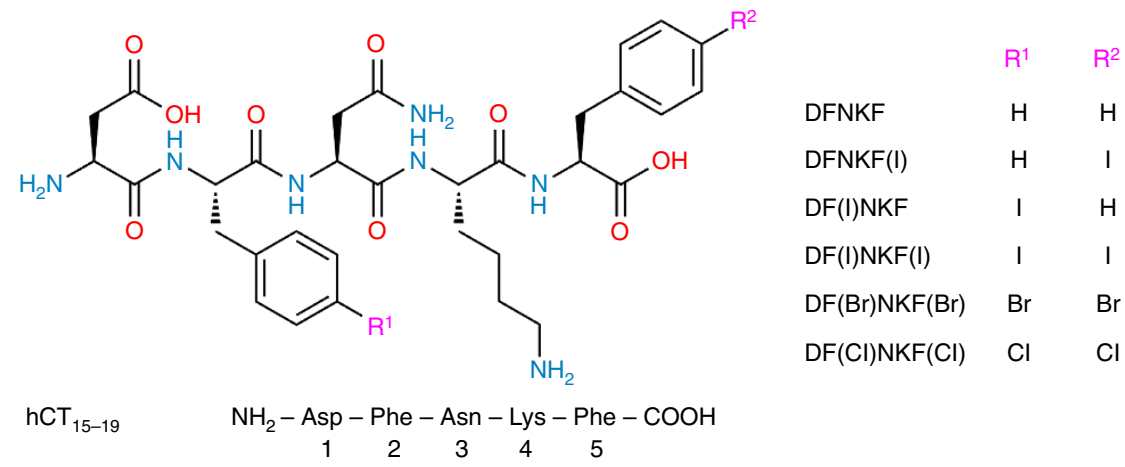

Figure 1 | Molecular formulae of the peptides used in this study. The natural peptide DFNKF was modified by halogenation on the phenylalanine residues in the para position. Modifications are shown in the peptide sequence in brackets where $F(I)$ denotes 4 -iodophenylalanine, $F(B r)$ denotes 4-bromophenylalanine and $\mathrm{F}(\mathrm{Cl})$ denotes 4-chlorophenylalanine. 
halogen bonds at the fibril strand surface to promote fibril-fibril lamination effects.

DFNKF is a short segment (residues 15-19) of the human calcitonin (hCT) hormone $\mathrm{e}^{25}$, an amyloidogenic protein with 32 amino acids, whose amyloid formation is related to medullary carcinoma of the thyroid ${ }^{24}$. This particular pentapeptide is widely used for investigating the nanoscopic arrangement of a fibril complex since DFNKF forms well-ordered fibrils similar to the aggregates of $\mathrm{hCT}^{24,25}$. For this reason, we selected DFNKF as a simple model compound for fibril formation and obtained its halogenated derivatives by introducing $\mathrm{I}, \mathrm{Br}$ and $\mathrm{Cl}$ atoms at the $p$-position of the phenylalanine (Phe) benzene ring. To study the effect on fibrillation of the introduction of halogen atoms on specific positions of the pentapeptide, we studied the 5 - $p$-iodoPhe derivative DFNKF(I), the 2-p-iodo-Phe derivative DF(I)NKF, the 2,5-bis- $p$-iodo-Phe derivative $\mathrm{DF}(\mathrm{I}) \mathrm{NKF}(\mathrm{I})$, as well as its bisbrominated and bis-chlorinated analogues $\mathrm{DF}(\mathrm{Br}) \mathrm{NKF}(\mathrm{Br})$ and $\mathrm{DF}(\mathrm{Cl}) \mathrm{NKF}(\mathrm{Cl})$, respectively (Fig. 1). Importantly, all of the studied peptides carry free amino $(\mathrm{N})$ and carboxyl $(\mathrm{C})$ termini.

Hydrogelation properties of halogenated DFNKF derivatives. Hydrogelation is highly indicative of fibrillation, therefore all peptides were in the first instance assessed for their ability to form hydrogels ${ }^{26,27}$. All of the studied peptides were found to selfassemble into hydrogels over a minimum gelation concentration. Only the hydrogel of the wild-type peptide DFNKF has been previously reported, though of its $\mathrm{N}$-acetylated form at the $\mathrm{N}$ terminus ${ }^{28}$. All the reported halogenated peptides are novel types of gelators and, importantly, formed gels (Fig. 2a and Supplementary Table 2) at concentrations much lower than that of their wild-type parent DFNKF, that is, $75 \mathrm{mM}$, with the supergelator peptide $\mathrm{DF}(\mathrm{I}) \mathrm{NKF}$ that showed a minimum gelation concentration of $<0.2 \%(2.5 \mathrm{mM}$; 30 -fold lower than DFNKF).

Attempts to study the kinetics of fibril formation using Thioflavin $\mathrm{T}$ (ThT) were unsuccessful since no increase in fluorescence was observed upon mixing ThT with peptide solutions. This is well in accordance with the literature since previous studies have demonstrated that DFNKF does not bind this fibril marker dye ${ }^{29}$.

Subsequently, a working concentration of $15 \mathrm{mM}$ was chosen where all halogenated peptides formed clear and elastic gels within a reasonable time $(\leq 12 \mathrm{~h})$. At this concentration, the speed of hydrogel formation followed the order DF(I)NK$\mathrm{F}(\mathrm{I})>\mathrm{DF}(\mathrm{I}) \mathrm{NKF} \geq \mathrm{DF}(\mathrm{Br}) \mathrm{NKF}(\mathrm{Br})>\mathrm{DF}(\mathrm{Cl}) \mathrm{NKF}(\mathrm{Cl}) \geq \mathrm{DFNKF}(\mathrm{I})$, whereas DFNKF did not form a gel even after 30 days (Supplementary Table 3). The diiodinated peptide formed the most homogeneous and shape-persistent gel (Fig. 2b), whereas peptides $\mathrm{DF}(\mathrm{Cl}) \mathrm{NKF}(\mathrm{Cl})$ and $\mathrm{DFNKF}(\mathrm{I})$ showed phase separation of the fibrous aggregates from the aqueous phase. The obtained hydrogels were all thermoreversible with gel-sol transition temperatures ranging from $65^{\circ} \mathrm{C}$ (DFNKF(I) to $119^{\circ} \mathrm{C}$ (DF(l)NKF(l); Fig. 2c). In particular, the observed transition temperatures followed the same order of the gel forming kinetics reported above. These results clearly show that, in general, halogenation of the DFNKF model peptide greatly promotes hydrogel formation, with the diiodinated peptide being the most efficient. This efficiency decreases with the polarizability of the halogen atom, that is, $\operatorname{DF}(\mathrm{l}) \mathrm{NKF}(\mathrm{l})>\mathrm{DF}(\mathrm{Br}) \mathrm{NKF}(\mathrm{Br})>$ $\mathrm{DF}(\mathrm{CI}) \mathrm{NKF}(\mathrm{CI})$, and also depends on the specific position of the halogen atom in the peptide scaffold, that is, the peptide iodinated at the Phe in the second position is more efficient than the one iodinated in the fifth.

Characterization of the different halogenated peptide hydrogels was done by oscillatory rheology (ring-cast method) using the $15 \mathrm{mM}$ concentration. At this low concentration, the wild-type peptide DFNKF only formed a viscous fluid and was not studied further. The diiodinated peptide $\mathrm{DF}(\mathrm{I}) \mathrm{NKF}(\mathrm{I})$ was confirmed to form the stiffest gel, which is reflected in its high elastic modulus $\left(\mathrm{G}^{\prime}>10^{4} \mathrm{~Pa}\right.$ and $\mathrm{G}^{\prime} \gg \mathrm{G}^{\prime \prime}$; Fig. 2d and Supplementary Fig. 3) and by the fact that the gel assumes the well-defined shape of the mould (Fig. 2b). The $\mathrm{G}^{\prime}$ value for $\mathrm{DF}(\mathrm{I}) \mathrm{NKF}(\mathrm{I})$ is comparable to
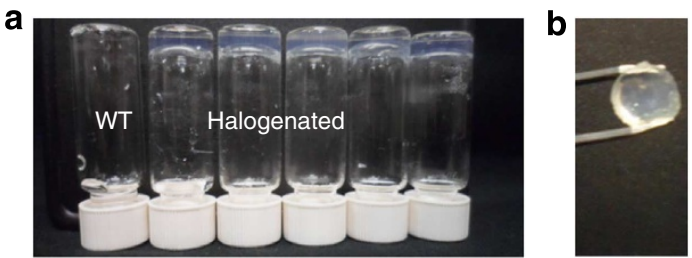

\begin{tabular}{cc} 
C & \\
\hline Peptide & Transition $T\left({ }^{\circ} \mathrm{C}\right)$ \\
\hline $\operatorname{DFNKF}(\mathrm{I})$ & $64.7-69.0$ \\
$\operatorname{DF}(\mathrm{I}) \mathrm{NKF}$ & $88.2-92.7$ \\
$\operatorname{DF}(\mathrm{I}) \mathrm{NKF}(\mathrm{I})$ & $114.0-119.0$ \\
$\operatorname{DF}(\mathrm{Br}) \operatorname{NKF}(\mathrm{Br})$ & $92.1-96.4$ \\
$\operatorname{DF}(\mathrm{Cl}) \operatorname{NKF}(\mathrm{Cl})$ & $78.1-82.4$ \\
\hline
\end{tabular}

\section{d}

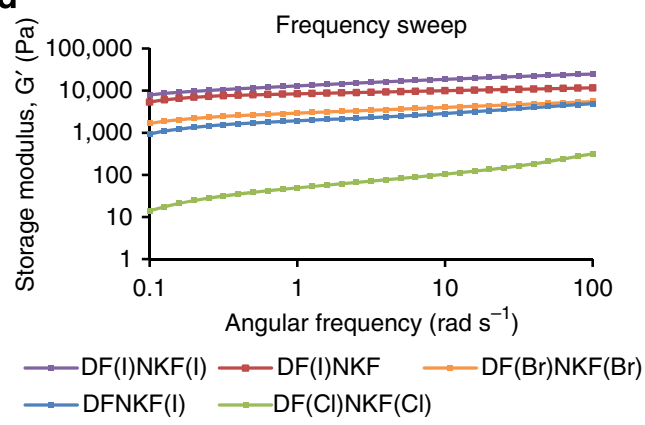

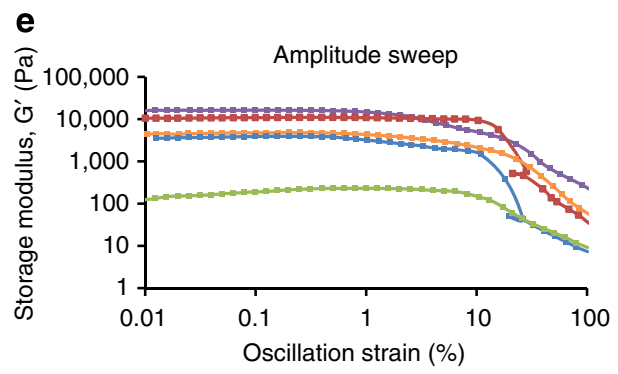

$\longrightarrow \mathrm{DF}(\mathrm{I}) \mathrm{NKF}(\mathrm{I}) \longrightarrow \mathrm{DF}(\mathrm{I}) \mathrm{NKF} \longrightarrow \mathrm{DF}(\mathrm{Br}) \mathrm{NKF}(\mathrm{Br})$
$\longrightarrow \mathrm{DFNKF}(\mathrm{I}) \longrightarrow \mathrm{DF}(\mathrm{Cl}) \mathrm{NKF}(\mathrm{Cl})$

Figure 2 | Characterization of hydrogels formed by halogenated DFNKF derivatives (a) Photograph of vials containing $15 \mathrm{mM}$ peptide solutions upon aging $12 \mathrm{~h}$ at RT After this period, all halogenated peptides formed clear hydrogels whereas the wild-type peptide DFNKF (WT) remained as a viscous fluid. (b) The hydrogel formed by $\operatorname{DF}(\mathrm{I}) \mathrm{NKF}(\mathrm{I})$ was the most shape-persistent of all gels. The gel was prepared using the ring-cast method at $15 \mathrm{mM}$ concentration and imaged after $48 \mathrm{~h}$. (c) Gel-sol transition temperatures of $15 \mathrm{mM}$ halogenated peptide hydrogels formed in $48 \mathrm{~h}$. (d) Rheological characterization of the gels by frequency sweep studies whereby the storage modulus $\left(G^{\prime}\right)$ was recorded as a function of angular frequency $(\omega)$. The peptide $\operatorname{DF}(\mathrm{I}) \mathrm{NKF}(\mathrm{I})$ was determined to be the strongest gel with $\mathrm{G}^{\prime}>10^{4} \mathrm{~Pa}$. (e) Amplitude sweep studies of peptide hydrogels showing $\mathrm{G}^{\prime}$ as a function of oscillation strain $(\gamma)$. All halogenated hydrogels show similar linear viscoelastic region (LVR) profiles. Peptide hydrogels at a concentration of $15 \mathrm{mM}$ were utilized for the rheological measurements after $48 \mathrm{~h}$ from preparation. For the loss moduli, see the Supplementary Fig. 3. 
that of Ac-LIVAGD, a hexamer that forms one of the best performing hydrogels in terms of ease of hydrogel formation and strength $^{30,31}$. Interestingly, the trend of $\mathrm{G}^{\prime}$ values parallels those of gel formation efficiency and thermal stability whereby gels of peptides $\mathrm{DF}(\mathrm{I}) \mathrm{NKF}(\mathrm{I}), \mathrm{DF}(\mathrm{I}) \mathrm{NKF}$ and $\mathrm{DF}(\mathrm{Br}) \mathrm{NKF}(\mathrm{Br})$ are the stiffest and DFNKF(I) and $\operatorname{DF}(\mathrm{Cl}) \operatorname{NKF}(\mathrm{Cl})$ formed the weakest ones.

Morphology and microarchitecture of the self-assembled fibrillar networks constituting the halogenated hydrogels were evaluated by confocal microscopy (Fig. 3) ${ }^{32}$. The network of the hydrogel of $\mathrm{DF}(\mathrm{I}) \mathrm{NKF}(\mathrm{I})$ appeared to be very filamentous with micrometre-long fibrils assembled in bundles with a helical sense, which propagate along the same direction. These micrometrelong fibrils become fewer and thinner on going from $\mathrm{DF}(\mathrm{I}) \mathrm{NKF}(\mathrm{I})$ to $\mathrm{DF}(\mathrm{I}) \mathrm{NKF}$ and $\mathrm{DF}(\mathrm{Br}) \mathrm{NKF}(\mathrm{Br})$, and disappear in the hydrogels of $\mathrm{DF}(\mathrm{CI}) \mathrm{NKF}(\mathrm{CI})$ and $\mathrm{DFNKF}(\mathrm{I})$ where only entangled matrices of small fibrils and aggregates were observed. These observations fit well with the rheology data where the strongest gels result from the entanglement of the longest fibrils. In agreement with the confocal microscopy study, the hydrogel formed by the peptide DF(I)NKF(I) showed an intertwined network of long fibrils in its transmission electron microscopy (TEM) image (Fig. 3f).

The nanostructure of the halogenated fibrils was revealed by atomic force microscopy (AFM) (Fig. 4). The peptides were first imaged after incubation in dilute aqueous conditions for 9 days (see also Supplementary Fig. 4) and Fig. 4a-c highlight the most strongly fibrillating peptides DF(I)NKF(I), DF(I)NKF and $\mathrm{DF}(\mathrm{Br}) \operatorname{NKF}(\mathrm{Br})$. While only small spherical aggregates were observed for the wild-type peptide DFNKF (Supplementary Fig. 4f), all of the halogenated peptides showed pronounced fibrillar structures. Large differences were, however, observed between the monoiodinated peptides DFNKF(I) and DF(I)NKF: Peptide DFNKF(I) (Supplementary Fig. 4e) showed only small protofibrils and aggregates, whereas the peptide DF(I)NKF (Fig. 4b and Supplementary Fig. 4c) showed large twisted fibrils similar to those of the diiodinated peptide DF(I)NKF(I) (Fig. 4a and Supplementary Fig. 4a), which, in turn, had the most complex structures with twisted ribbon-like fibrils tens of nanometre wide and micrometre long. These results highlight the importance of the specific position of the iodine atom in the peptide structure and therefore the subsequent specific interactions, which result in different amyloid fibril topographies. That the fibrillation and twisting is not due to drying is excluded by high-resolution cryo-TEM and electron tomography (ET) studies. This is exemplified for the peptide $\mathrm{DF}(\mathrm{I}) \mathrm{NKF}(\mathrm{I})$ corresponding to the strongest hydrogel. It shows fibrils with lateral sizes of $20-25 \mathrm{~nm}$ and helical twisting with periodicities between 250 and $140 \mathrm{~nm}$ (Fig. 4h and Supplementary Fig. 4b). Such periodicities correspond to the different maturation steps of amyloid fibrils as previously reported for $\beta$-lactoglobulin fibrils ${ }^{33}$. ET allowed three-dimensional visualization of the twisting morphology of a DF(I)NKF(I) fibril imaged in solution (Fig. 4i).

Amyloid structure of fibrils of halogenated peptides. There are three criteria that define a protein aggregate as an amyloid fibril: green birefringence upon staining with Congo Red, fibrillar morphology and $\beta$-sheet secondary structure ${ }^{34}$. Therefore, Congo red staining was carried out for all the peptides (at the same $15 \mathrm{mM}$ concentration) and strong green-gold birefringence was observed under polarized light, which is a reporter for long-range cross- $\beta$ structure (Supplementary Fig. 5).

Further evidence of amyloid nature was obtained by Fourier transform infrared (FT-IR) spectroscopy. The FT-IR spectra of both the solution and the gel state in $\mathrm{D}_{2} \mathrm{O}$ were recorded (Supplementary Fig. 6). A solution of the wild-type peptide DFNKF showed no significant amide I' band, however, using a concentration at which it forms a gel $(75 \mathrm{mM})$, this band became visible. This peak at around $1,630 \mathrm{~cm}^{-1}$ can be attributed to the peptide bond carbonyl upon formation of the amyloid $\beta$-sheet structure and subsequent gelation ${ }^{35}$. The spectra of the gel state
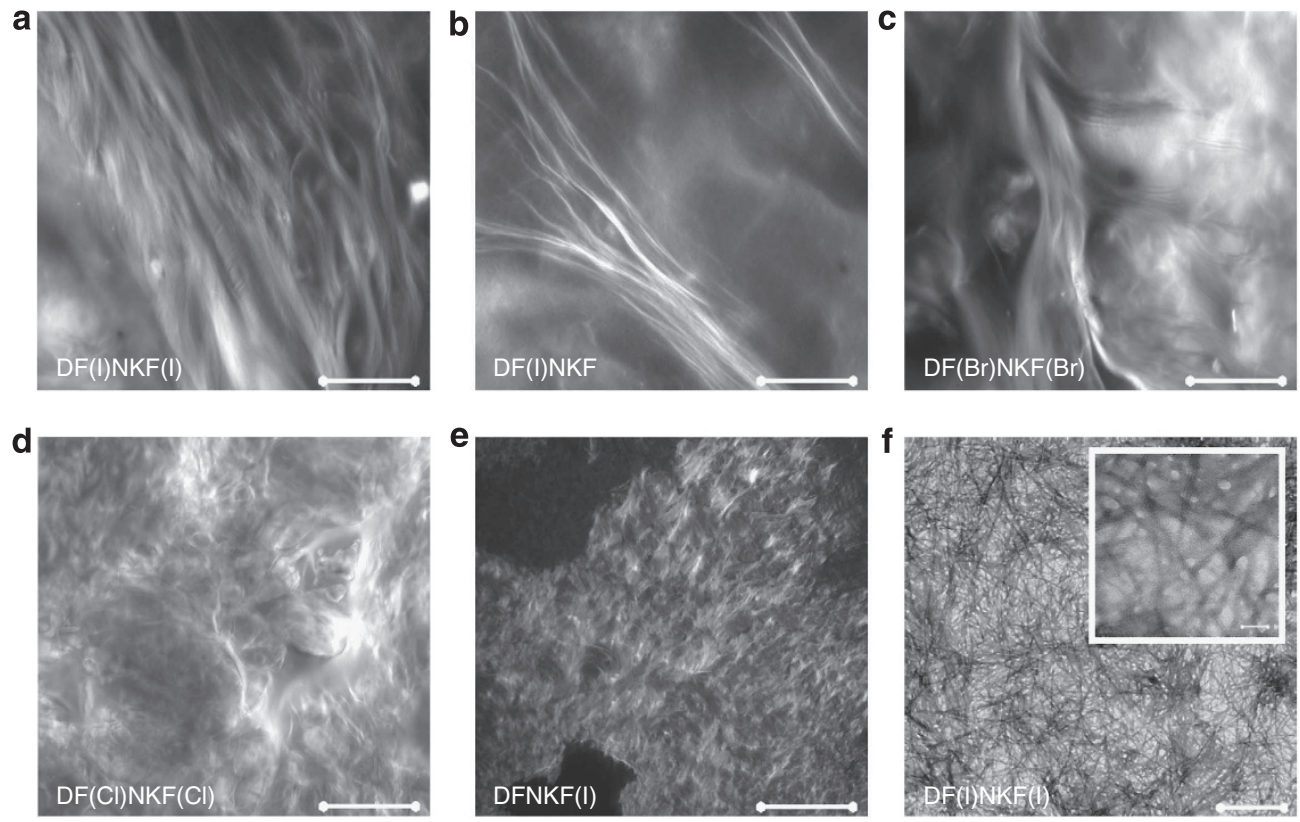

Figure 3 | Fibrillar morphologies of halogenated hydrogels. (a-e) Confocal microscopy images of peptide hydrogels at a concentration of $15 \mathrm{mM}$ upon aging $48 \mathrm{~h}$ at $\mathrm{RT}$ and after staining with Rhodamine $\mathrm{B}$ (scale bar, $100 \mu \mathrm{m}$ ). Panels $\mathbf{a}, \mathbf{b}$ and $\mathbf{c}$ show bundles of long twisted fibrils belonging to hydrogels of peptides $\operatorname{DF}(\mathrm{I}) \operatorname{NKF}(\mathrm{I}), \mathrm{DF}(\mathrm{I}) \mathrm{NKF}$ and $\mathrm{DF}(\mathrm{Br}) \operatorname{NKF}(\mathrm{Br})$, respectively. Panels $\mathbf{d}$ and $\mathbf{e}$ show much smaller fibrils of hydrogels of peptides $\mathrm{DF}(\mathrm{Cl}) \mathrm{NKF}(\mathrm{Cl})$ and DFNKF(I), which intertwine and form a matrix-type structure. (f) TEM image of the dried hydrogel of peptide DF(I)NKF(I) showing an intertwined network of long fibrils (scale bars, $0.5 \mu \mathrm{m}$ and $5 \mathrm{~nm}$ ). 
a

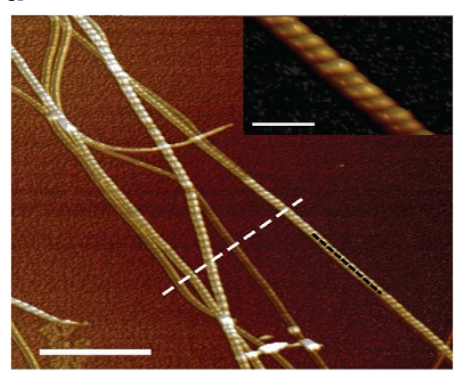

d

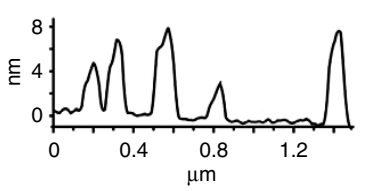

g

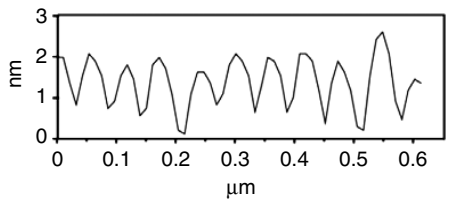

i

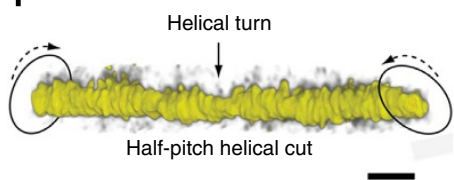

b

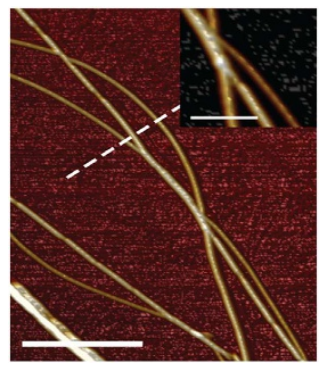

e

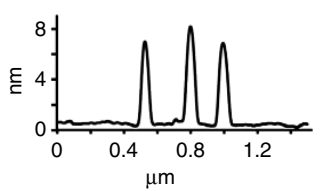

h

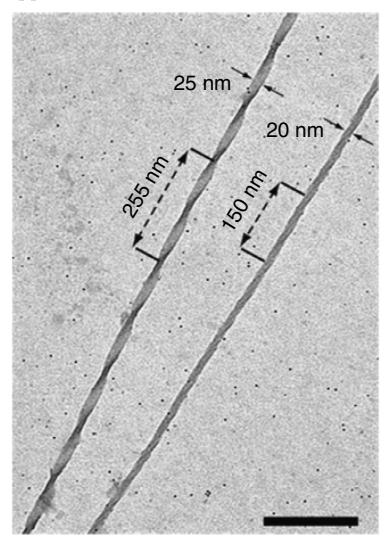

C
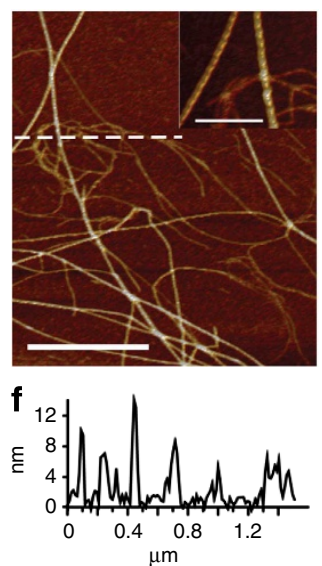

$\mu \mathrm{m}$

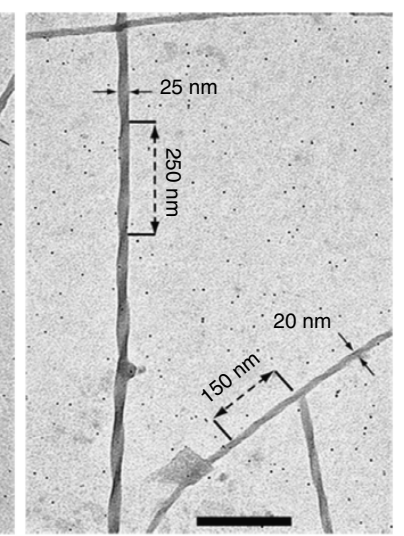

Figure 4 | Nanofibrillation of the halogenated pentapeptides. AFM image close-up insets of (a) peptide DF(I)NKF(I), (b) peptide DF(I)NKF, and (c) peptide $\operatorname{DF}(\mathrm{Br}) \mathrm{NKF}(\mathrm{Br})$ evaporated on mica substrates after 9 days incubation in aqueous solutions (scale bar, $1 \mu \mathrm{m}$ ). (d-f) Height profiles of fibrils from a, $\mathbf{b}$ and $\mathbf{c}$ (cross-section lines highlighted in white). (g) Cross-sectional analysis on the top of a fibril segment in a showing a helicoidal profile along the longitudinal direction of the fibrils (highlighted in black). (h) Cryo-TEM images of the dried hydrogel of DF(I)NKF, showing a network of long fibrils (scale bar, $200 \mathrm{~nm}$ ). (i) In situ electron tomography of half-pitch helicoidal peptide DF(I)NKF(I) vitrified from aqueous solution. The twisted fibril morphology is highlighted by arrows. Electron tomography reconstructions were collected upon tilting of samples up to $\pm 69^{\circ}$ (scale bar, $25 \mathrm{~nm}$ ).

of the halogenated peptides showed this same amide I' band consistent with the formation of amyloid fibrillar species comprising $\beta$-sheet elements. These data confirm that all the studied halogenated derivatives of DFNKF form fibrils of the same amyloid nature to their wild-type parent. Interestingly, this $1,630 \mathrm{~cm}^{-1}$ peak is red-shifted in the spectra of DF(I)NKF(I) and DF(I)NKF, while it is not in the one of DFNKF(I) (Supplementary Fig. 6). This red-shift indicates a reduced electron density on the peptide bond and is consistent with the involvement of the carbonyl oxygen in halogen bonding ${ }^{36}$.

Raman spectroscopy is a powerful tool to investigate the occurrence of the halogen bond ${ }^{37}$. For this reason, we followed the changes experienced by the C-I stretching band at around $167 \mathrm{~cm}^{-1}$ upon formation of the gel. Interestingly, compared with the bulk powders of the starting materials, in the dried gels of the iodinated peptides DF(I)NKF(I) and DF(I)NKF, this band shifted to lower frequency (see Supplementary Fig. 7). This shift is perfectly consistent with the weakening of the C-I bond as a consequence of its involvement in halogen bonding upon formation of the hydrogels ${ }^{37}$.

Co-assembly of wild-type peptide with diiodinated analogue. Bromination and chlorination of proteins in vivo has been related to a series of oxidative stress-related diseases such as cystic fibrosis $^{38}$, atherosclerotic intima ${ }^{39}$, sepsis ${ }^{40}$ and asthma, among others $^{41}$. A direct correlation exists between the role of oxidative stress and protein fibrillation ${ }^{42}$. However, how halogenation affects protein structure, folding and functioning is not yet known. To study to what extent a partial degree of halogenation may promote the amplified formation of amyloid fibrils, we studied the co-assembly ${ }^{43}$ of the strongly self-assembling diiodinated peptide $\mathrm{DF}(\mathrm{I}) \mathrm{NKF}(\mathrm{I})$ with the wild-type peptide DFNKF. Mixing DFNKF and DF(I)NKF(I) at concentrations at which normally neither form gels alone (Fig. 5a), 15 and $0.75 \mathrm{mM}$ (5\%), respectively, resulted in a mixed hydrogel within $18 \mathrm{~h}$.

Characterization of the physical properties of the mixed hydrogel was performed by oscillatory rheology (ring-cast method) where the mixed hydrogel was found to be weaker than the hydrogel containing only $\mathrm{DF}(\mathrm{I}) \mathrm{NKF}(\mathrm{I})$ (Fig. $5 \mathrm{~b}$ versus Fig. 2d). It was also observed that increasing the amount of the strong gelator $\mathrm{DF}(\mathrm{I}) \mathrm{NKF}(\mathrm{I})$ led to an increase in the elastic modulus value, whereas the gel containing $10 \%$ was stronger than the one containing $5 \%$ of $\mathrm{DF}(\mathrm{I}) \mathrm{NKF}(\mathrm{I})$. Interestingly, circular dichroism (CD) analysis of the mixed hydrogels showed the same structural features observed in the CD spectra of the pure iodinated peptides (see Supplementary Figs 8 and 9).

The resulting fibril morphologies in the mixed hydrogels were studied by AFM (Fig. 5c,d). A $15 \mathrm{mM}$ sample of the wild-type peptide DFNKF showed only a mixture of intertwined aggregates and small fibrils, which is consistent with the observation that no gelation is observed at this concentration since the fibrils appear 
a

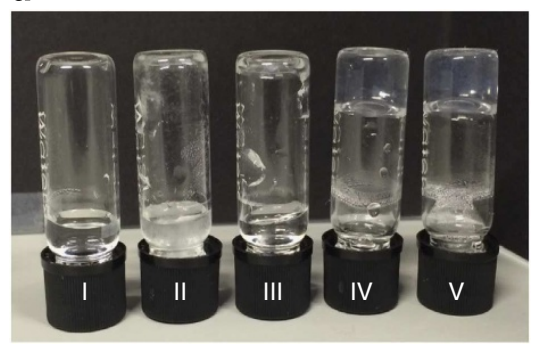

C

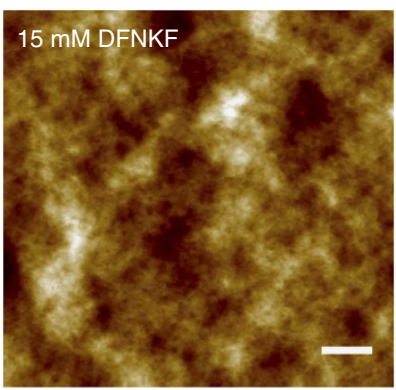

b

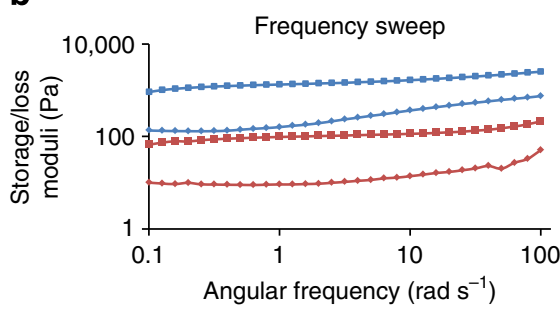

$\longrightarrow 10 \% \mathrm{DF}(\mathrm{I}) \mathrm{NKF}(\mathrm{I}) \mathrm{G}^{\prime} \longrightarrow 10 \% \mathrm{DF}(\mathrm{I}) \mathrm{NKF}(\mathrm{I}) \mathrm{G}^{\prime \prime}$ $\longrightarrow 5 \% \mathrm{DF}(\mathrm{I}) \mathrm{NKF}(\mathrm{I}) \mathrm{G}^{\prime} \longrightarrow 5 \% \mathrm{DF}(\mathrm{I}) \operatorname{NKF}(\mathrm{I}) \mathrm{G}^{\prime \prime}$

d

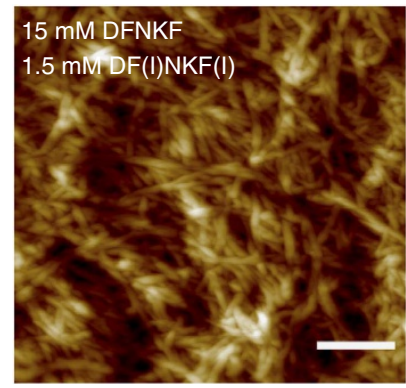

Figure 5 | Mixed hydrogels formed by co-assembly of DFNKF and DF(I)NKF(I). (a) Photograph of vials after $48 \mathrm{~h}$ containing (I) $15 \mathrm{mM}$ DFNKF, which did not form hydrogel; (II) $1.5 \mathrm{mM} \mathrm{DF(I)NKF(I),} \mathrm{which} \mathrm{did} \mathrm{not} \mathrm{form} \mathrm{hydrogel} \mathrm{and} \mathrm{some} \mathrm{precipitation} \mathrm{was} \mathrm{observed;} \mathrm{(III)} 15 \mathrm{mM}$ DFNKF:0.15 mM $\operatorname{DF}(\mathrm{I}) \mathrm{NKF}(\mathrm{I})$, which did not form hydrogel; (IV) $15 \mathrm{mM}$ DFNKF:0.75 mM DF(I)NKF(I), which formed hydrogel within $48 \mathrm{~h}$; (V) $15 \mathrm{mM}$ DFNKF:1.5 mM $\operatorname{DF}(I) N K F(I)$, which formed hydrogel within $48 \mathrm{~h}$. (b) Rheological characterization of mixed hydrogels by frequency sweep studies whereby the storage concentration of $15 \mathrm{mM}$ after $48 \mathrm{~h}$ were utilized for the rheological measurements. (c) AFM of a $15 \mathrm{mM}$ solution of wild-type peptide DFNKF showed small fibrils and aggregates unable to network sufficiently to form a hydrogel. (d) AFM of a sample of the mixed hydrogel containing $15 \mathrm{mM}$ DFNKF and 1.5 mM DF(I)NKF(I) showed larger and more entangled fibrils (scale bar, $400 \mathrm{~nm}$ ).

too small to crosslink and form a network (Fig. 5c). On the addition of $10 \% \mathrm{DF}(\mathrm{I}) \mathrm{NKF}(\mathrm{I})$, the resulting fibrils were much longer and intertwined to form a fibrous network resulting in the hydrogelation of the wild-type peptide DFNKF solution (Fig. $5 \mathrm{~d}$ and Supplementary Fig. 10a). The weakness of the resulting mixed hydrogel compared with that of $\mathrm{DF}(\mathrm{I}) \mathrm{NKF}(\mathrm{I})$ alone can be explained by the relative size and network formation of the fibrils (Supplementary Fig. 10b). This result may have important implications in a biological setting since only small amounts of brominated or chlorinated peptides may be required to amplify the supramolecular self-assembly of an amyloid fibril.

Role of halogen bonding in hydrogels' self-assembly. Although no direct structural evidence of halogen bonding between our peptide monomers has been obtained, yet, the reported results, IR and Raman in particular, provided corroborative evidence that iodinated peptides $\mathrm{DF}(\mathrm{I}) \mathrm{NKF}$ and $\mathrm{DF}(\mathrm{I}) \mathrm{NKF}(\mathrm{I})$ potentially use halogen bonds to efficiently self-assemble into mature amyloid fibrils. In fact, the particular position of the iodinated residue in the peptide sequence greatly influences the efficiency of its selfassembly process, thus suggesting the involvement of the iodine atom in a specific noncovalent interaction (Fig. 6a), rather than nonspecific hydrophobic and/or steric effects. This is further supported by considerations of halogen atom polarizability since the dibrominated and dichlorinated derivatives $\mathrm{DF}(\mathrm{Br}) \mathrm{NKF}(\mathrm{Br})$ and $\mathrm{DF}(\mathrm{Cl}) \mathrm{NKF}(\mathrm{Cl})$ showed less tendency to form fibrils than their diiodinated analogue $\mathrm{DF}(\mathrm{I}) \mathrm{NKF}(\mathrm{I})$. This result allows interactions involving halogen atoms as hydrogen bond acceptors to be ruled out as $\mathrm{Cl}$ and $\mathrm{Br}$ would give stronger interactions than I, which was not observed. Electrostatic interactions involving the ionizable side chains of the peptides, Asp and Lys, may also be ruled out as the formation of these halogenated hydrogels is largely $\mathrm{pH}$-independent (see Supplementary Table 4). As a
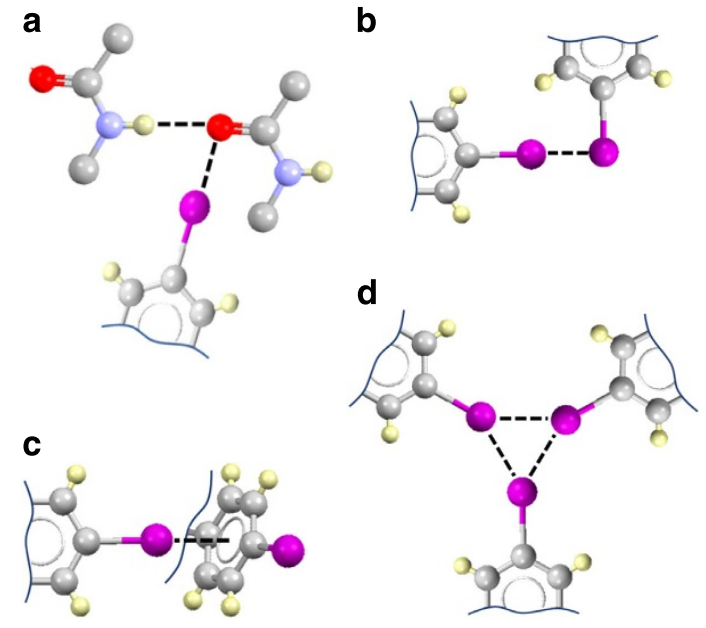

Figure 6 | Potential halogen bonds involving p-iodo-phenylalanine. IR and Raman spectroscopies are more consistent with the occurrence of type a interaction, however, other interactions reported cannot be discounted. (a) Orthogonal hydrogen and halogen bonds involving the carbonyl oxygen atom of the peptide bond ${ }^{36}$. (b) Type II iodine . . iodine contacts ${ }^{23}$. (c) lodine $\cdots \pi$ interactions ${ }^{49}$. (d) Triangular iodine synthon ${ }^{50}$.

confirmation of the minor role played by electrostatic attraction in the self-assembly of the reported halogenated peptides, increasing the ionic strength of the peptide solutions resulted in accelerated formation of the hydrogels (see Supplementary Table 5). It is also unlikely that aromatic $\pi-\pi$ interactions are playing an important role in the self-assembly of the halogenated hydrogels, since this would not explain the polarizability dependence that we observed. Finally, hydrophobic interactions could not justify the observed trends in hydrogel properties because 
$\mathrm{DF}(\mathrm{Br}) \mathrm{NKF}(\mathrm{Br})$ and $\mathrm{DF}(\mathrm{Cl}) \mathrm{NKF}(\mathrm{Cl})$ are both more hydrophobic than DF(I)NKF (see Supplementary Table 6), which instead forms the second stiffest gel. Moreover, the two monoiodinated peptides, despite having similar hydrophobicity, display completely different properties. For the same reasons discussed above, electrostatic and hydrophobic interactions also should not play an important role in the interfibrillar association observed in the halogenated gels.

\section{Discussion}

In summary, we have demonstrated that iodination of a short amyloidogenic core sequence strongly promotes its fibril formation ability and affects the structure of formed fibrils. In particular, the 2-p-iodo-Phe derivative DF(I)NKF was demonstrated to be 30 -fold more efficient in forming hydrogels than the wild-type pentapeptide DFNKF. This is remarkable for such a modest perturbation in structure. Preliminary results indicate that the strong effect of iodination on peptide selfassembly is general and not limited to the core sequence reported here. In fact, iodination of the amyloidogenic core sequence KLVFF (residues 16-20 of A $\beta$ ) as well as of the full-length hCT similarly promotes fibril formation efficiency and self-assembly (data not shown). Iodination of aromatic amino acids may thus develop as a general strategy for the design of new hydrogels starting from unprotected peptides and without the use of organic solvents ${ }^{44,45}$.

The results reported in this paper are relevant in the field of the programmed synthesis of amyloid supramolecular assemblies as well as in the context of amyloid-dependent diseases. In the former field, we have demonstrated that single atom mutations of amyloidogenic core sequences by replacing hydrogen with iodine atoms greatly promote fibril formation and self-assembly. Methods promoting the formation of mature fibrils are advantageous for use, among others, as scaffolds for tissue engineering, controlled drug release, surgical reconstruction applications, microfluidic devices, biosensors and bioswitches ${ }^{7-11}$. As far as amyloid-dependent diseases are concerned, our results suggest that oxidative stress-induced halogenation of proteins might potentially be the triggering point for the transformation of a natively folded protein into a halogenbonded and fibrillar malfunctioning form. Research in this direction is currently being carried out in our laboratories and will be reported elsewhere.

\section{Methods}

Reagents. Congo red, Rhodamine $\mathrm{B}, \mathrm{NaCl}$ and $\mathrm{D}_{2} \mathrm{O}$ were purchased from SigmaAldrich and used without further purification. Peptides with confirmed amino acid analysis (purity $\geq 98 \%$ ), were purchased from Biopeptek (Malvern, USA). The integrity of all peptides was confirmed by ion spray mass spectrometry and the purity was determined by reverse phase high-pressure liquid chromatography.

Hydrogel preparation. Hydrogels were prepared by dissolving each peptide ( $15 \mathrm{mM}$ for halogenated peptides and $75 \mathrm{mM}$ for the wild-type peptide DFNKF) in deionized water $(18.2 \mathrm{M \omega} \mathrm{cm})$ or in $\mathrm{D}_{2} \mathrm{O}$ ( $\geq 99.9$ atom \% deuterium). The glass vials containing the $500 \mu \mathrm{l}$ solutions were sealed, sonicated for $20 \mathrm{~s}$, heated using a heat gun until complete dissolution of the peptides, before slow cooling to room temperature (RT) All the samples were stored at RT for $48 \mathrm{~h}$ before analysis.

Preparation of peptide solutions. Peptide solutions $(40 \mu \mathrm{M})$ were freshly prepared in deionized water $(18.2 \mathrm{M \omega cm})$, sonicated for $20 \mathrm{~s}$, and gently warmed to reach $90{ }^{\circ} \mathrm{C}$ before filtration through a $0.22 \mu \mathrm{m}$ Millipore filter. The peptide solutions were stored in sealed vials at RT for varying time points before analysis.

X-ray crystallography. The $p$-iodo-phenylalanine was suspended in water at RT and kept in an open vial under a hood. After 6 months, colourless crystalline needles appeared. Suitable crystals for XRD measurements were analysed without further manipulation. The crystals were measured using Mo-K $\alpha$ radiation on a Bruker KAPPA APEX II diffractometer with a Bruker KRYOFLEX low temperature device. The crystal structure was solved by direct method and refined against $\mathrm{F}^{2}$ using SHELXL97 ${ }^{46}$. Packing diagrams were generated using Mercury ${ }^{47}$. The non-hydrogen atoms were refined anisotropically and hydrogen atoms were fixed geometrically and refined isotropically (CCDC deposition number: 1049844).

Mixed gels preparation. Mixed hydrogels were prepared using the same method as previously described. Briefly, peptides were weighed into a glass vial and MilliQ water added. The glass vial was sealed and sonicated for $20 \mathrm{~s}$ before heating with a heat gun until complete solubilization of the peptides. The vials were allowed then to cool slowly to RT and stored at RT for 7 days before analysis.

Thermal stability. The vials containing the hydrogels at $15 \mathrm{mM}$ were inverted and fixed at the bottom of an oil bath with stirring. The temperature was kept at $25^{\circ} \mathrm{C}$ for $10 \mathrm{~min}$ to equilibrate the system and then gradually increased from 25 to $140^{\circ} \mathrm{C}$ $\left(\right.$ at $1^{\circ} \mathrm{C} \mathrm{min}{ }^{-1}$ ). The temperature at which the gels break is reported as a range: the initial temperature corresponds to the fall of the first drop and the final one to the complete breakdown of the gel.

Rheology. Rheology experiments were performed using a TA instrument ARG2 Rheometer. A $20 \mathrm{~mm}$ stainless steel, parallel-plate geometry was used with a gap distance of $1,000 \mu \mathrm{m}$. Oscillatory frequency sweep studies were performed for a range of $0.1-100 \mathrm{rad} \mathrm{s}^{-1}$, using a $0.5 \%$ strain. Oscillatory amplitude sweep studies were conducted from 0.01 to $100 \%$ strain with an angular frequency of $1 \mathrm{rads}^{-1}$. The ring-cast method was used for hydrogel preparation at a peptide concentration of $15 \mathrm{mM}$. The peptide solution was sonicated for $20 \mathrm{~s}$ in a sealed glass vial before heating to $90^{\circ} \mathrm{C}$ to afford complete dissolution of the peptide. After cooling, the solutions were transferred into ring casts of $22 \mathrm{~mm}$ diameter and placed in tightly sealed tissue-culture dishes for $48 \mathrm{~h}$. All the measurements were repeated a minimum of three times.

Circular dichroism spectroscopy. All the CD experiments were carried out in deionized water $(18.2 \mathrm{M} \Omega \mathrm{cm})$ in a $0.01 \mathrm{~mm}$ detachable quartz cuvette, using a JASCO J-815 CD spectrometer. Acquisitions were performed between 190 and $300 \mathrm{~nm}$ with a $0.1 \mathrm{~nm}$ data pitch, $1 \mathrm{~nm}$ bandwidth, $100 \mathrm{~nm} \mathrm{~min}^{-1}$ scanning speed and $1 \mathrm{~s}$ response time. All the spectra are an average of 10 scans and were corrected from a reference solution, comprising deionized water $(18.2 \mathrm{M} \Omega . \mathrm{cm})$ alone. Raw data $(\theta$, in millidegree) were subsequently converted to mean residue ellipticity $\left([\theta]\right.$ in $\left.\operatorname{deg} \mathrm{cm}^{2} \mathrm{dmol}^{-1}\right)$ for the sake of comparison, in accordance with the following formulae:

$$
[\theta]=\frac{\theta}{10 \times l \times c \times(n-1)},
$$

where $\theta$ is the observed ellipticity in millidegree, $c$ is the concentration of the sample in moll ${ }^{-1},(n-1)$ is the number of peptide bonds and $l$ is the path length of the cuvette in centimetre.

Confocal microscopy. Hydrogels were imaged using a Zeiss LSM 710 microscope with a He/Ne laser $\left(\lambda_{\mathrm{ex}}=543 \mathrm{~nm}\right)$. The fluorescent dye, Rhodamine B, was incorporated into an aged hydrogel $(48 \mathrm{~h})$ scaffold by the addition of $10 \mu \mathrm{l}$ of the dye solution $(0.1 \% \mathrm{w} / \mathrm{v})$. Following complete absorption of the dye, the sample was excited at $543 \mathrm{~nm}$ and emitted light recorded using the E570LP emission filter.

Congo red staining. All the samples were monitored for green birefringence using an Olympus BX50 polarizing microscope with a SensiCam PCO camera used to display and enhance images. An $80 \%$ ethanol solution saturated with $\mathrm{NaCl}$ and Congo red was freshly prepared before each measurement. A piece of each peptide hydrogel was placed on a glass microscope, allowed to air dry and then stained with Congo red solution. Subsequently, excess Congo red solution was blotted off the slide and the samples were analysed using both bright and polarized light.

Infrared spectroscopy (FT-IR). Infrared spectra were recorded at RT using a Nicolet iS50 FT-IR spectrometer equipped with a DTGS detector. Peptides were analysed as solutions (after heating at $100^{\circ} \mathrm{C}$ to break any pre-formed fibrils) or gels at $15 \mathrm{mM}$ in $\mathrm{D}_{2} \mathrm{O}$ (for DFNKF $75 \mathrm{mM}$ concentration was used). Spectra represent an average of 64 scans recorded in a single-beam mode with a $4 \mathrm{~cm}^{-1}$ resolution and corrected for the background. The second-derivative analyses of the spectra were performed using the Nicolet FTIR software, Omnic 9.0, with a 13-point and third polynomial order Savitzky and Golay function. Secondderivative spectra generated negative bands as compared with the original spectra, thus for comparison all the second-derivative spectra were multiplied by -1 .

Raman spectroscopy. Raman spectra were acquired at RT by using a Horiba Xplora MicroRaman instrument equipped with an Olympus BX-41 Microscope. An excitation wavelength of $785 \mathrm{~nm}$ was used. Laser power was attenuated by

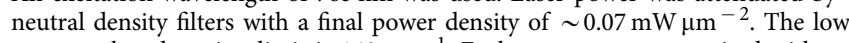
wavenumber detection limit is $140 \mathrm{~cm}^{-1}$. Each spectrum was acquired with an exposure time of $5 \mathrm{~s}$ over 35 cycles. The raw data were first corrected from the baseline, using the JASCO Nicolet FTIR software, Omnic 9.0, between 140 
and $340 \mathrm{~cm}^{-1}$. Obtained data were subsequently normalized, for the sake of comparison, and plotted using Origin Pro 8.

Atomic force microscopy. Peptide solution $(10 \mu \mathrm{l}, 40 \mu \mathrm{M})$ or diluted gel was deposited onto freshly cleaved mica surface and air dried. AFM characterizations were performed on a Veeco Dimension 5,000 Scanning Probe Microscope with a Nanoscope V controller (Digital Instruments, Inc.). All the samples were prepared on mica substrate and measured without treatment. Al-coated silicon AFM tips (NSC 15/AIBS, MikroMasch, Estonia) with a tip radius of $10 \mathrm{~nm}$ were used to probe the surface profiles of the films. Tapping-mode AFM imaging was used according to well-established procedures. All the images were post-treated with NanoScope Analysis 1.5 Software.

Transmission electron microscopy. High-resolution transmission electron microscopy imaging was carried out using JEM-3200Fsc field emission microscope (JEOL) operated at $300 \mathrm{kV}$ in bright-field mode with Omega-type Zero-loss energy filter. The images were acquired with ULTRASCAN 4,000 CCD camera (GATAN) and with GATAN DIGITAL MICROGRAPH software, while the specimen temperature was maintained at $-187^{\circ} \mathrm{C}$. Dried TEM and ET samples were prepared by placing $4 \mu \mathrm{l}$ of $40 \mu \mathrm{M} \mathrm{DF}(\mathrm{I}) \mathrm{NKF}(\mathrm{I})$ solution on 200 mesh carbon only grid $(\mathrm{CFT} 200-\mathrm{Cu})$ and the excess removed with filter paper.

Electron tomography. For image alignment purposes, the TEM grids were dipped in 11-mercapto-1-undecanol ligand-coated gold particle solution $(d=3-10 \mathrm{~nm})$ before sample deposition. ET tilt series were acquired with the SERIALEMsoftware package between tilt angles of $\pm 69^{\circ}$. Prealignment of tilt image series was done with IMOD and the fine alignment and cropping with JPEGANIM software package ${ }^{48}$. The images were binned twice to reduce noise and computation time. Maximum entropy method reconstruction scheme was carried out with MEM software package ${ }^{48}$ on Linux cluster with regularization parameter value of $\delta=5.0 \times 10^{-2}$. Data visualization, volumetric graphics and analyses were performed with the UCSF CHIMERA package. The tomogram was filtered with CHIMERA's Gaussian filter with 1.5 voxel width.

\section{References}

1. Knowles, T. P. J. \& Buehler, M. J. Nanomechanics of functional and pathological amyloid materials. Nat. Nanotechnol. 6, 469-479 (2011).

2. Selkoe, D. J. Alzheimer's disease: genes, proteins, and therapy. Physiol. Rev. 81, 741-766 (2001).

3. Dobson, C. M. Protein folding and misfolding. Nature 426, 884-890 (2003).

4. Selkoe, D. J. Folding proteins in fatal ways. Nature 426, 900-904 (2003).

5. Chiti, F. \& Dobson, C. M. Protein misfolding, functional amyloid and human disease. Annu. Rev. Biochem. 75, 333-366 (2006).

6. Kreplak, L. \& Aebi, U. From the polymorphism of amyloid fibrils to their assembly mechanism and cytotoxicity. Adv. Protein Chem. 73, 217-233 (2006).

7. Hauser, C. A. E., Maurer-Stroh, S. \& Martins, I. C. Amyloid-based nanosensors and nanodevices. Chem. Soc. Rev. 43, 5326-5345 (2014).

8. Adamcik, J. \& Mezzenga, R. Fibrils from a polymer physics perspective. Macromolecules 45, 1137-1150 (2012).

9. Li, C. \& Mezzenga, R. The interplay between carbon nanomaterials and amyloid fibrils in bio-nanotechnology. Nanoscale 5, 6207-6218 (2013).

10. Mankar, S., Anoop, A., Sen, S. \& Maji, S. K. Nanomaterials: amyloids reflect their brighter side. Nano Rev. 2, 6032-6043 (2011).

11. Cherny, I. \& Gazit, E. Amyloids: not only pathological agents but also ordered nanomaterials. Angew. Chem. Int. Ed. 47, 4062-4069 (2008).

12. Griffith Jones, O. \& Mezzenga, R. Inhibiting, promoting, and preserving stability of functional protein fibrils. Soft Matter 8, 876-895 (2012).

13. Petkova, A. T. et al. A structural model for Alzheimer's $\beta$-amyloid fibrils based on experimental constraints from solid state NMR. Proc. Natl Acad. Sci. USA 99, 16742-16747 (2002).

14. Metrangolo, P. \& Resnati, G. Halogen versus hydrogen. Science 321, 918-919 (2008).

15. Ryan, D. M., Anderson, S. B., Senguen, F. T., Youngman, R. E. \& Nilsson, B. L. Self-assembly and hydrogelation promoted by F5-phenylalanine. Soft Matter 6, 475-479 (2010).

16. Wang, Y., Zhang, Z., Xu, L., Li, X. \& Chen, H. Hydrogels of halogenated Fmoc-short peptides for potential application in tissue engineering. Colloids Surf. B Biointerfaces 104, 163-168 (2013).

17. Reches, M. \& Gazit, E. Designed aromatic homo-dipeptides: formation of ordered nanostructures and potential nanotechnological applications. Phys. Biol. 3, S10-S19 (2006).

18. Metrangolo, P., Pilati, T. \& Resnati, G. Halogen bonding and other noncovalent interactions involving halogens: a terminology issue. Cryst. Eng. Comm. 8, 946-947 (2006).

19. Ryan, D. M., Doran, T. M., Anderson, S. B. \& Nilsson, B. L. Effect of C-terminal modification on the self-assembly and hydrogelation of fluorinated Fmoc-Phe derivatives. Langmuir 27, 4029-4039 (2011).
20. Ryan, D. M., Anderson, S. B. \& Nilsson, B. L. The influence of side-chain halogenation on the self-assembly and hydrogelation of Fmoc-phenylalanine derivatives. Soft Matter 6, 3220-3231 (2010).

21. Desiraju, G. R. et al. Definition of the halogen bond (IUPAC Recommendations 2013). Pure Appl. Chem. 85, 1711-1713 (2013).

22. Metrangolo, P. \& Resnati, G. Tracing iodine. Nat. Chem. 3, 260 (2011).

23. Metrangolo, P. \& Resnati, G. Type II halogen . . halogen contacts are halogen bonds. IUCrJ 1, 5-7 (2014).

24. Berger, G., Berger, N., Guillaud, M. H., Trouillas, J. \& Vauzelle, J. L. Calcitonin-like immunoreactivity of amyloid fibrils in medullary thyroid carcinomas. An immunoelectron microscope study. Virchows Arch. A Pathol. Anat. Histopathol. 412, 543-551 (1988).

25. Arvinte, T., Cudd, A. \& Drake, A. F. The structure and mechanism of formation of human calcitonin fibrils. J. Biol. Chem. 268, 6415-6422 (1993).

26. Steed, J. W. Supramolecular gel chemistry: developments over the last decade Chem. Commun. 47, 1379-1383 (2011)

27. Meazza, L. et al. Halogen-bonding-triggered supramolecular gel formation. Nat. Chem. 5, 42-47 (2013).

28. Lakshmanana, A. et al. Aliphatic peptides show similar self-assembly to amyloid core sequences, challenging the importance of aromatic interactions in amyloidosis. Proc. Natl Acad. Sci. USA 110, 519-524 (2013).

29. Shtainfeld, A., Sheynis, T. \& Jelinek, R. Specific mutations alter fibrillation kinetics, fiber morphologies, and membrane interactions of pentapeptides derived from human calcitonin. Biochemistry 49, 5299-5307 (2010).

30. Hauser, C. A. E. et al. Natural tri- to hexapeptides self-assemble in water to amyloid $\beta$-type fiber aggregates by unexpected $\alpha$-helical intermediate structures. Proc. Natl Acad. Sci. USA 108, 1361-1366 (2011).

31. Mishra, A. et al. Ultrasmall natural peptides self-assemble to strong temperature-resistant helical fibers in scaffolds suitable for tissue engineering. Nano Today 6, 232-239 (2011)

32. Smith, D. K. In Supramolecular Chemistry: From Molecules to Nanomaterials (John Wiley and Sons, Inc., 1999).

33. Adamcik, J. et al. Understanding amyloid aggregation by statistical analysis of atomic force microscopy images. Nat. Nanotechnol. 5, 423-428 (2010).

34. Nilsson, M. R. Techniques to study amyloid fibril formation in vitro. Methods 34, 151-160 (2004).

35. Shivu, B. et al. Distinct $\beta$-sheet structure in protein aggregates determined by ATR-FTIR spectroscopy. Biochemistry 52, 5176-5183 (2013).

36. Vasylyeva, V. et al. Orthogonal halogen and hydrogen bonds involving a peptide bond model. Cryst. Eng. Comm. 16, 8102-8105 (2014).

37. Messina, M. T. et al. Infrared and Raman analyses of the halogen-bonded non-covalent adducts formed by $\alpha$ - $\varpi$-diiodoperfluoroalkanes with DABCO and other electron donors. J. Mol. Struct. 524, 87-94 (2000).

38. Vliet, V. D. et al. Myeloperoxidase and protein oxidation in cystic fibrosis. Am J. Physiol. Lung Cell. Mol. Physiol. 279, L537-L546 (2000).

39. Hazen, S. L. 3-chlorotyrosine, a specific marker of myeloperoxidase? Catalyzed oxidation is markedly elevated in low density lipoprotein isolated from human atherosclerotic intima. J. Clin. Invest. 99, 2075-2075 (1997).

40. Gaut, J. P. Neutrophils employ the myeloperoxidase system to generate antimicrobial brominating and chlorinating oxidants during sepsis. Proc. Natl Acad. Sci. USA 98, 11961-11961 (2001).

41. $\mathrm{Wu}, \mathrm{W}$. et al. Eosinophils generate brominating oxidants in allergen-induced asthma. J. Clin. Invest. 105, 1455-1463 (2000).

42. Mazzulli, J. R., Hodara, R., Lind, S. \& Ischiropoulos, H. Oxidative stress and protein deposition diseases in protein misfolding, aggregation, and conformational diseases. Prot. Rev. 4, 123-133 (2006).

43. Ryan, D. M., Doran, T. M. \& Nilsson, B. L. Complementary $\pi-\pi$ interactions induce multicomponent coassembly into functional fibrils. Langmuir. 27, 11145-11156 (2011).

44. Gazit, E. Searching sequence space. Nat. Chem. 7, 14-15 (2015).

45. Frederix, P. W. J. M. et al. Exploring the sequence space for (tri-)peptide selfassembly to design and discover new hydrogels. Nat. Chem. 7, 30-37 (2015)

46. Sheldrick, G. M. A short history of SHELX. Acta Crystallogr. Sect. A 64, $112-122$ (2008).

47. Macrae, C. F. et al. Mercury CSD 2.0 - new features for the visualization and investigation of crystal structures. J. Appl. Cryst. 41, 466-470 (2008).

48. Engelhardt, P. Electron tomography of chromosome structure. Encyclopedia of analytical chemistry (2006).

49. Sirimulla, S., Bailey, J. B., Vegesna, R. \& Narayan, M. Halogen interactions in protein-ligand complexes: implications of halogen bonding for rational drug design. J. Chem. Inf. Model. 53, 2781-2791 (2013).

50. Lu, Y. et al. Triangular halogen trimers. A DFT study of the structure, cooperativity, and vibrational properties. J. Phys. Chem. A 109, 11956-11961 (2005).

\section{Acknowledgements}

The European Research Council is acknowledged for the Starting Grant ERC-2012 StG_20111012 FOLDHALO (Grant Agreement Number 307108) to P.M. Dr Claudia Pigliacelli (Politecnico di Milano) is acknowledged for help with the TEM experiment of Fig. $3 \mathrm{f}$. 


\section{Author contributions}

A.B. and L.P. performed most of the peptide self-assembly work. L.S. performed the IR spectroscopy and ionic strength-pH-dependence experiments. N.H. measured the samples by AFM. J.S.H. measured the samples by TEM. L.C. and G.T. performed the single crystal X-ray analysis. G.G. and L.V. performed the Raman spectroscopy. P.M., R.M., O.I., and G.R. conceived the experiments and contributed to results discussion. All the authors contributed to manuscript writing.

\section{Additional information}

Accession codes: The X-ray crystallographic coordinates for the structure reported in this study have been deposited at the Cambridge Crystallographic Data Centre (CCDC), under deposition number 1049844. These data can be obtained free of charge from The Cambridge Crystallographic Data Centre via www.ccdc.cam.ac.uk/data_request/cif.

Supplementary Information accompanies this paper at http://www.nature.com/ naturecommunications
Competing financial interests: The authors declare no competing financial interests.

Reprints and permission information is available online at http://npg.nature.com/ reprintsandpermissions/

How to cite this article: Bertolani, A. et al. Supramolecular amplification of amyloid self-assembly by iodination. Nat. Commun. 6:7574 doi: 10.1038/ncomms8574 (2015).

(c) (i) This work is licensed under a Creative Commons Attribution 4.0 International License. The images or other third party material in this article are included in the article's Creative Commons license, unless indicated otherwise in the credit line; if the material is not included under the Creative Commons license, users will need to obtain permission from the license holder to reproduce the material. To view a copy of this license, visit http://creativecommons.org/licenses/by/4.0/ 